\title{
Nanoporous Waveguide Spectroscopy for the Estimation of Enzyme Adsorption on Mesoporous Silica
}

\author{
Hiroyuki Arafune, ${ }^{* 1 \dagger}$ Kazuhiro Hotta, ${ }^{* 2}$ Tetsuji Itoh, ${ }^{* 3}$ Norio Teramae, ${ }^{* 2}$ and Akira Yamaguchi ${ }^{* 4 *}$ \\ *1 Department of Creative Engineering, National Institute of Technology, Tsuruoka College, 104 Sawada, \\ Inooka, Tsuruoka 997-8511, Japan \\ *2 Department of Chemistry, Graduate School of Science, Tohoku University, Aoba, Sendai 980-8578, Japan \\ *3 Research Center for Compact Chemical System, National Institute of Advanced Industrial Science and \\ Technology (AIST), 4-2-1 Nigatake, Miyagino, Sendai 983-8551, Japan \\ *4 College of Science, Ibaraki University, 2-1-1 Bunkyo, Mito, Ibaraki 310-8512, Japan
}

\begin{abstract}
Mesoporous silica is considered as promising host material for enzymes due to its uniform pore size of enzyme dimensions and tunable surface chemical properties. In this study, we applied nanoporous waveguide (NPWG) spectroscopy to observe adsorption dynamics of heme proteins with different molecular size within mesoporous silica film modified with different surface functional groups. Since NPWG spectroscopy provides kinetic information and rough quantification of adsorption amount, it is useful to study the adsorption process of enzymes within inorganic nanoporous materials.
\end{abstract}

Keywords Enzyme adsorption, waveguide spectroscopy, mesoporous silica, adsorption dynamics, heme proteins

(Received February 13, 2017; Accepted February 22, 2017; Published April 10, 2017)

\section{Introduction}

An enzyme has highly selective and efficient catalytic abilities under mild reaction conditions, and it has been used as an important biocatalyst for food processing, biosensors, drugs and so on. ${ }^{1}$ Since the activity of enzymes dissolved in a bulk aqueous solution is easily degraded, studies have been conducted to achieve long-term stability of their activity. Enzyme immobilization inside inorganic nanopores is a simple and effective way to maintain catalytic activity. ${ }^{2-4}$ Surfactanttemplated mesoporous silica is a powerful candidate for an ideal host of biomolecules due to its uniform pore size of dimensions of biomolecules and tunable surface chemical properties. ${ }^{4-6}$ The size-matching between the enzyme and pore size has been proposed to stabilize structure and function of the enzyme immobilized within mesoporous silica. The enzyme within mesoporous silica can be easily recovered from a reaction solution. Until now, various biocatalytic systems based on the mesoporous silica host have been studied.

Adsorption of enzymes into porous media is usually studied by batch process, which is monitored by a decrease in the amount of enzyme in a solution phase. ${ }^{7}$ The batch process can be used to study kinetics of slow adsorption process, while their application for observing rapid adsorption process is difficult. Label-free and high-throughput optical observation techniques have hence been developed for the study of adsorption kinetics of enzymes at porous media. For example, Chen et al. applied interferometric spectroscopy for studying adsorption dynamics

$\dagger$ To whom correspondence should be addressed.

E-mail: harafune@tsuruoka-nct.ac.jp (H. A.); akira.yamaguchi. sci@vc.ibaraki.ac.jp (A.Y.) of bovine serum albumin, hemoglobin and myoglobin on a porous silicon substrate. ${ }^{8}$ The microscopic method has also been applied for a single-particle system. ${ }^{9,10}$ Optical waveguide spectroscopy is one of the sensitive methods for a label-free and high-throughput method to study rapid adsorption kinetics. ${ }^{11-14}$ In our previous studies, a mesoporous film on an $\mathrm{Al}$ thin layer was used for a planar waveguide, and its waveguiding properties could be used to analyze rapid adsorption kinetics of proteins. ${ }^{13,14}$ Hereafter, optical waveguide spectroscopy based on the nanoporous/aluminum multilayer film is called as nanoporous waveguide (NPWG) spectroscopy.

In the present study, we applied NPWG spectroscopy to characterize the adsorption dynamics of heme proteins (cytochrome $c$, myoglobin, horse radish peroxidase) at a mesoporous silica film with pore size of $c a .6 \mathrm{~nm}$. The heme proteins used in this study have different isoelectric point $(\mathrm{pI})$ and molecular size. NPWG spectroscopy utilizes optical waveguide modes excited in mesoporous silica/Al multilayer film, which is observed as a sharp attenuation dip in the reflection spectrum (Fig. 1). Since the position of an attenuation dip corresponds to the change of a refractive index of a mesoporous silica layer, a molecular adsorption process into silica mesopores can be observed by monitoring a shift of the attenuation dip. Herein, the adsorption of these heme proteins at the mesoporous silica layer was studied to discuss the effects of a molecular size and the surface-protein interaction for the adsorption process. In addition, effects of surface modification of mesoporous silica on the adsorption process were also studied. 


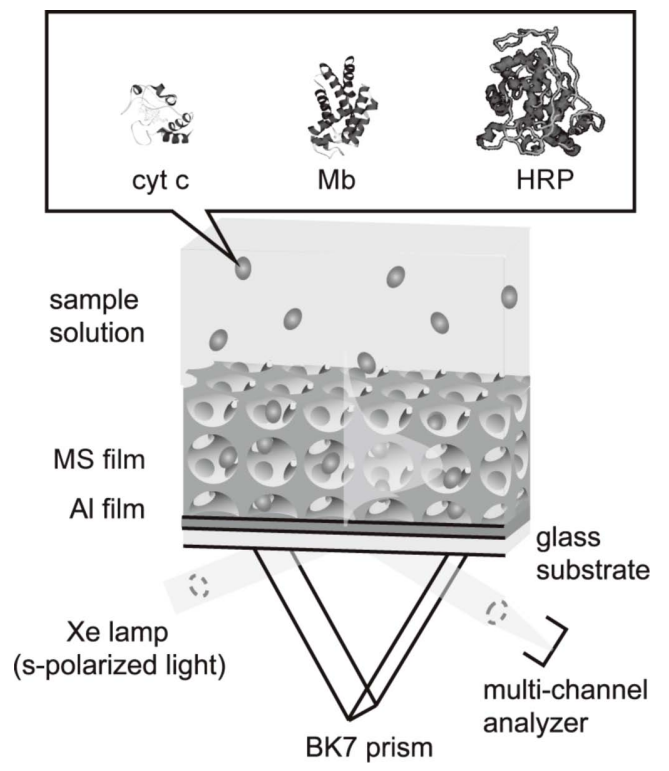

Fig. 1 Schematic illustration of a configuration of NPWG spectroscopy and an idealized electric distribution of the TE0 mode.

\section{Experimental}

\section{Reagents and chemicals}

Cytochrome $c$ (cyt $c$ ) from equine heart, myoglobin $(\mathrm{Mb})$ from horse heart, horse radish peroxidase (HRP) and non-ionic triblock copolymer Pluronic F127 (F127: PEO $_{106} \quad$ PPO $_{70}$ $\mathrm{PEO}_{106}$ ) consisting of polyethylene oxide (PEO) and polypropylene oxide (PPO) were purchased from Sigma-Aldrich (Tokyo, Japan). A rectangular cover glass substrate $(24 \times 45 \times$ $0.3 \mathrm{~mm}$, Matsunami Glass Ind., Ltd., Osaka, Japan) was used for each sample substrates. Al wire (99.99\%; Nilaco Co., Tokyo, Japan) was used for the thermal deposition of an Al layer on the glass substrate. Milli-Q water was used for all experiments. Other chemicals were purchased from Wako Pure Chemical Industries, Ltd. (Osaka, Japan).

\section{Preparation of MS film}

Precursor solution for the mesoporous silica (MS) layer was prepared according to our previous report. ${ }^{14}$ A mixture of Pluronic F127 (1.38 g), 1 M hydrochloric acid solution ( $0.30 \mathrm{~g})$, ethanol $(26.49 \mathrm{~g})$, and water $(2.07 \mathrm{~g})$ was stirred for $1 \mathrm{~h}$ at $30^{\circ} \mathrm{C}$. Then, tetraethoxysilane (TEOS, $3.0 \mathrm{~g}$ ) was added to the mixture and the mixed solution was stirred for $2 \mathrm{~h}$ at $30^{\circ} \mathrm{C}$ to give the precursor solution.

The glass substrate was sonicated in acetone, methanol, and then Milli-Q water for $5 \mathrm{~min}$ each. Then it was immersed in concentrated nitric acid and boiled for $75 \mathrm{~min}$ to remove organic species adsorbed on the substrate surface. Finally, its surface was etched by immersing it in a $10 \mathrm{wt} \%$ hydrofluoric acid solution for $20 \mathrm{~s}$. An aluminum layer $(12 \mathrm{~nm})$ was deposited on the one side of the cleaned-glass substrate using an Ulvac model VPC-1100 vacuum deposition system. The MS layer was dipcoated on the Al-deposited glass substrate using the precursor solution of mesoporous silica. The sample substrate with the MS/Al multilayer film was heated at $70^{\circ} \mathrm{C}$ overnight, and then, it was calcined at $420^{\circ} \mathrm{C}$ for $4 \mathrm{~h}$ to remove the template surfactant. We designated the mesoporous silica layer after calcination as Cal-MS. Thickness of Cal-MS was $320 \mathrm{~nm}$.
The inner pore surface of Cal-MS was modified with ethoxy group or octadecylsilyl (ODS) group to study the effect of surface moiety on adsorption dynamics of enzymes. Surface treatment of mesoporous silica with ethanol to modifying ethoxy group on silica surface was according to the literature. ${ }^{15}$ Briefly, Cal-MS/Al was refluxed in dry ethanol $(20 \mathrm{~mL})$ at $80^{\circ} \mathrm{C}$ for $16 \mathrm{~h}$, and then dried under vacuum for $4 \mathrm{~h}$ to obtain ethoxymodified MS/Al (Ethoxy-MS/Al). ODS modified mesoporous silica film (ODS-MS) was obtained by refluxing Cal-MS/Al in toluene $(20 \mathrm{~mL})$ containing octadecyldimethylchlorosilane for $1 \mathrm{~h}$, followed by end-capping of residual silanol groups with trimethylchlorosilane. ${ }^{14}$

\section{NPWG spectroscopy}

The details of the experimental setup for the OWG spectroscopy were described in our previous report. ${ }^{16}$ In brief, s-polarized white light from a Xe lamp (Hamamatsu Photonics; L8254, 150W) was irradiated on the MS/Al sample substrate and its reflection spectra were measured by using a multichannel analyzer (Hamamatsu Photonics; PMA-11). A fluidic channel (total volume, $2.8 \mu \mathrm{L}$; channel width, $1.5 \mathrm{~mm}$; channel depth, $0.14 \mathrm{~mm}$ ) was fabricated using a PDMS substrate that was placed on the MS/Al/glass sample substrate. A syringe pump (Harvard apparatus; Model 11 plus) was used to deliver the sample solution into the fluidic channel and the OWG spectroscopy measurements were performed with continuous flow of enzymes in a phosphate buffer solution $(\mathrm{pH} 5.0,6.0$ or $7.0)$ at $15 \mu \mathrm{L} \mathrm{min}{ }^{-1}$. Ionic strength of each buffer solution was set as $0.01 \mathrm{M}$.

\section{Results and Discussion}

The position of the attenuation peak shifts toward longer wavelength due to the adsorption of enzymes within MS layer. The wavelength shift of the attenuation peak $\left(\Delta \lambda_{\text {owG }}\right)$ in the reflection spectrum is approximately proportional to the amount of adsorbent within a mesoporous film as given in Eq. (1), ${ }^{14}$

$$
\Delta \lambda_{\mathrm{oWG}}=a q_{\mathrm{ads}}
$$

where $a$ is a proportional constant and $q_{\text {ads }}$ is the amount of adsorbent. $\Delta \lambda_{\text {owG }}$ thus can be used to monitor the relative amount of enzyme adsorbed within the MS layer. The increase and decrease of $\Delta \lambda_{\text {owg }}$ are associated with adsorption and desorption of enzymes, respectively. On the basis of Fick's low, time-course shift of $\lambda_{\text {owG }}, \Delta \lambda_{\text {owG }}(t)$, can be described as follows: ${ }^{14}$

$$
\Delta \lambda_{\mathrm{owG}}(t)=\Delta \lambda_{\mathrm{owG}}(\infty)\left(1-\frac{8}{\pi^{2}} \sum_{n=0}^{\infty} \frac{\exp \left[(2 n+1)^{2} D_{\mathrm{app}} t / l^{2}\right]}{(2 n+1)^{2}}\right)
$$

where $\Delta \lambda_{\text {owG }}(\infty), D_{\text {app }}$, and $l$ are the $\Delta \lambda_{\text {owg }}$ value at equilibrium, the apparent diffusion coefficient (in $\mathrm{cm}^{2} \mathrm{~s}^{-1}$ ) of enzyme inside the mesopores, and the thickness of the porous layer (in $\mathrm{cm}$ ), respectively. The Eq. (2) was used to analyze the adsorption kinetics of enzymes.

We observed adsorption behaviors of heme proteins under a continuous flow of each protein solution (100 $\mu \mathrm{M}, \mathrm{pH} 6.0)$. Adsorption profiles of cyt $c, \mathrm{Mb}$ and HRP at Cal-MS are shown in Fig. 2. These profiles could be well-fitted to Eq. (2), and the values of $\Delta \lambda_{\text {owG }}(\infty)$ and $D_{\text {app }}$ are summarized in Table 1 . It is clearly recognized that both $\Delta \lambda_{\text {owG }}(\infty)$ and $D_{\text {app }}$ values are lower for larger protein; cyt $c>\mathrm{Mb}>\mathrm{HRP}$. Since $\mathrm{pI}$ values of $\mathrm{Mb}$ and HRP are almost the same, it can be considered that $\mathrm{Mb}$ and 


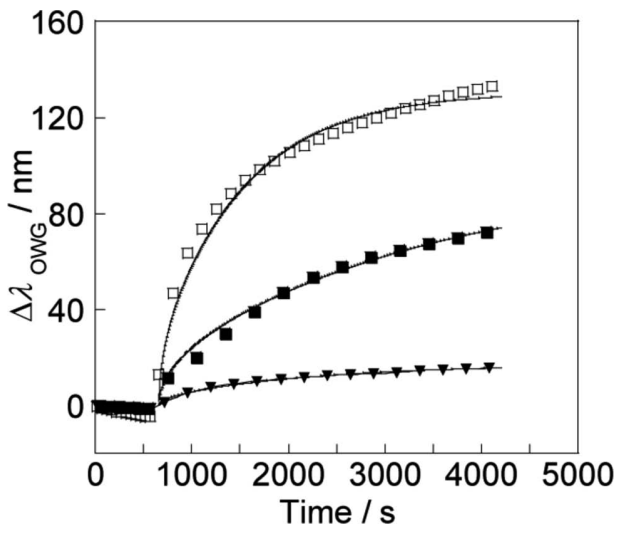

Fig. 2 Adsorption profiles of cyt $c$ (open square), $\mathrm{Mb}$ (filled square) and HRP (filled triangle) under the flow of a $100 \mu \mathrm{M}$ solution (pH 6.0) at silica mesopores. The solid lines are the results of the best fit using Eq. (2).

Table 1 Physical properties of each enzymes and calculated values of $\Delta \lambda_{\text {owG }}(\infty)$ and $D_{\text {app }}$ molecular size of enzymes are based on existing literature ${ }^{5}$

\begin{tabular}{lccccc}
\hline Protein & $\begin{array}{c}\text { Size/ } \\
\mathrm{nm}\end{array}$ & $\begin{array}{c}\mathrm{Mw} / \\
\mathrm{kDa}\end{array}$ & $\mathrm{pI}$ & $\begin{array}{c}\Delta \lambda_{\mathrm{OWG}}(\infty) / \\
\mathrm{nm}\end{array}$ & $\begin{array}{c}D_{\text {app }} / \\
\mathrm{cm}^{2} \mathrm{~s}^{-1}\end{array}$ \\
\hline cyt $c$ & $2.6 \times 3.2 \times 3.3$ & 12 & 10.7 & 134 & $15 \times 10^{-13}$ \\
$\mathrm{Mb}$ & $2.1 \times 3.5 \times 4.4$ & 17 & $7.0-7.3$ & 96 & $5.1 \times 10^{-13}$ \\
$\mathrm{HRP}$ & $4.0 \times 4.4 \times 6.8$ & 44 & 7.2 & 17 & $0.6 \times 10^{-13}$ \\
\hline
\end{tabular}

HRP exhibited similar protein-protein interaction and proteinsurface interaction. The lowest $D_{\text {app }}$ for HRP is hence supposed to be due to the spatial restriction. The longest axis of HRP is $6.8 \mathrm{~nm}$, which is almost consistent with the pore diameter of Cal-MS ( $c a .6 \mathrm{~nm})$. This size-matching would induce the slowest diffusivity of HRP inside the silica mesopore. Since cyt $c$ and $\mathrm{Mb}$ are smaller than the mesopore diameter, less contribution of the spatial restriction for the diffusion of those proteins is expected.

The diffusion of small proteins (cyt $c$ and $\mathrm{Mb}$ ) was further studied by adsorption dynamics depending on solution $\mathrm{pH}$. Figure 3(a) shows adsorption profiles of cyt $c$ and $\mathrm{Mb}$ observed under different $\mathrm{pH}$ conditions. The $\Delta \lambda_{\text {owg }}$ and $D_{\text {app }}$ values estimated from those adsorption profiles are summarized in Table 2. For cyt $c, \Delta \lambda_{\text {owg }}$ and $D_{\text {app }}$ tend to be larger as $\mathrm{pH}$ increased, suggesting that the increase in the adsorbed amount of cyt $c$ was related to the acceleration of cyt $c$ mobility inside the pore. Keeping in mind the pI of cyt $c$ (10.7), positive surface charge of cyt $c$ was almost the same at pH 5.0-7.0. By contrast, dissociation constants of surface silanol group are 4.5 and $8.5,{ }^{17}$ where the negative surface charge of the inner pore surface significantly increased with increasing $\mathrm{pH}$ from 5.0 and 7.0. It can be considered that the electrostatic interaction between cyt $c$ and the silica surface became larger with increasing $\mathrm{pH} .{ }^{18}$ The strong electrostatic interaction led to the increase in the initial concentration of cyt $c$ at the top surface of Cal-MS layer accompanied by rapid diffusion into the pore interior by a concentration gradient. In addition, an electrostatic repulsion between charged $\mathrm{Mb}$ molecules might contribute to the diffusivity of $\mathrm{Mb}$ inside the pore. $\Delta \lambda_{\text {owG }}(\infty)$ and $D_{\text {app }}$ values of $\mathrm{Mb}$ show relatively high value at $\mathrm{pH}$ 6.0. Since $\mathrm{Mb}$ is neutral or weakly charged in this $\mathrm{pH}$ region, the obtained result is

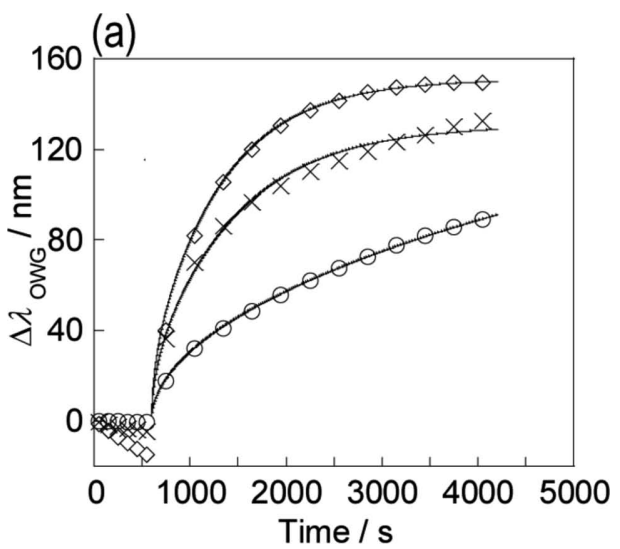

(b)

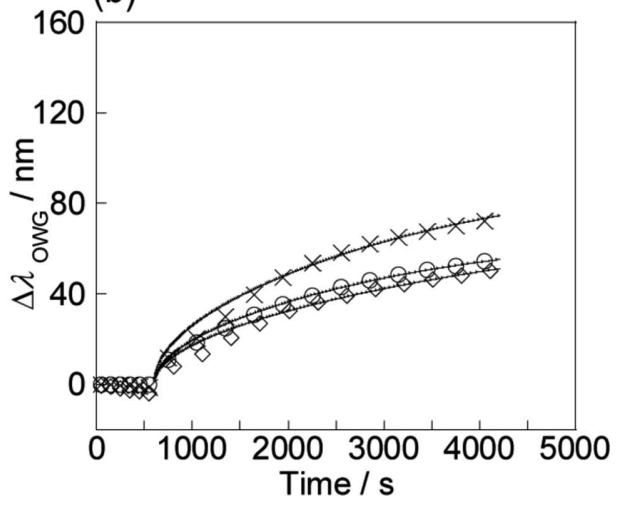

Fig. 3 Adsorption profiles of (a) cyt $c$ and (b) Mb under the flow of a $100 \mu \mathrm{M}$ solution with various $\mathrm{pH}$ conditions at silica mesopore $(\bigcirc$, $\mathrm{pH} 5.0 ; \times, \mathrm{pH} 6.0 ; \diamond, \mathrm{pH} 7.0)$. The solid lines are the results of the best fit using Eq. (2).

Table 2 Calculated values of $\Delta \lambda_{\text {owG }}(\infty)$ and $D_{\text {app }}$ of cyt $c$ and $\mathrm{Mb}$ at various $\mathrm{pH}$

\begin{tabular}{cccccc}
\hline & \multicolumn{3}{c}{ cyt $c$} & & \multicolumn{2}{c}{$\mathrm{Mb}$} \\
\cline { 2 - 3 } \cline { 5 - 6 } $\mathrm{pH}$ & $\begin{array}{c}\Delta \lambda_{\text {OWG }}(\infty) / \\
\mathrm{nm}\end{array}$ & $\begin{array}{c}D_{\text {app }} / \\
\mathrm{cm}^{2} \mathrm{~s}^{-1}\end{array}$ & & $\begin{array}{c}\Delta \lambda_{\text {owG }}(\infty) / \\
\mathrm{nm}\end{array}$ & $\begin{array}{c}D_{\text {app }} / \\
\mathrm{cm}^{2} \mathrm{~s}^{-1}\end{array}$ \\
\hline 5.0 & 91 & $3.0 \times 10^{-13}$ & & 69 & $4.7 \times 10^{-13}$ \\
6.0 & 134 & $15 \times 10^{-13}$ & & 96 & $5.1 \times 10^{-13}$ \\
7.0 & 150 & $18 \times 10^{-13}$ & & 65 & $3.4 \times 10^{-13}$ \\
\hline
\end{tabular}

supposed to be due to the complementary effect of the decrease in electrostatic repulsion between $\mathrm{Mb}-\mathrm{Mb}$ and electrostatic attraction between Mb-silica. Diffusivity of $\mathrm{Mb}$ was remarkably smaller than that of cyt $c$ at above $\mathrm{pH}$ 6.0, whereas the relation is reversed at $\mathrm{pH}$ 5.0. If the molecular size governs the diffusivity of proteins inside the silica mesopore, diffusivity of small cyt $c$ would be larger regardless of the solution $\mathrm{pH}$. Therefore, it is considered that the diffusivity of those heme proteins is likely governed by the protein-surface electrostatic interaction rather than the molecular size.

The influence of hydrophobicity of the inner pore surface on the adsorption process was examined by comparing adsorption behaviors of $\mathrm{Mb}$ at Cal-MS, Ethoxy-MS, and ODS-MS layers. Figure 4(a) shows typical reflection spectra for the Ethoxy-MS layer before/after $\mathrm{Mb}$ adsorption. The adsorption profiles of $\mathrm{Mb}$ at each MS layers are summarized in Fig. 4(b). The order of $\Delta \lambda_{\text {owG }}(\infty)$ is Ethoxy-MS $>$ Cal-MS $>$ ODS-MS, indicating that 


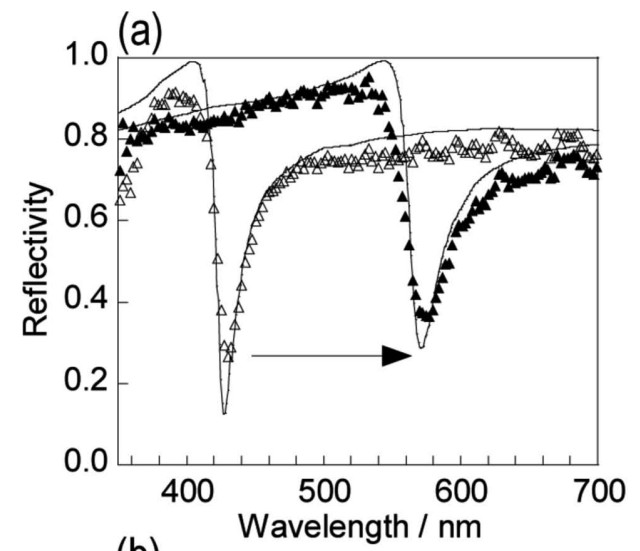

(b)

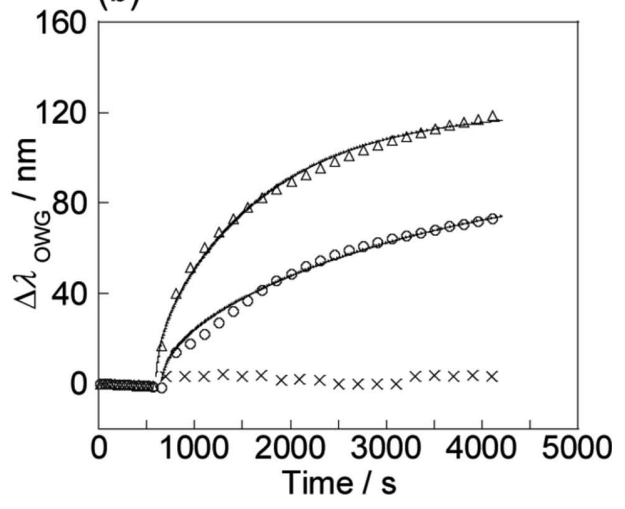

Fig. 4 (a) Reflection spectra of Ethoxy-MS before (open triangle) and after (shaded triangle) under the flow of a $100 \mu \mathrm{M} \mathrm{Mb}$ solution ( $\mathrm{pH}$ 6.0) and calculated spectra (lines) obtained by Fresnel calculation. (b) Adsorption dynamics of $100 \mu \mathrm{M} \mathrm{Mb}$ solution (pH 6.0) at EthoxyMS (triangle), Cal-MS (circle) and ODS-MS (cross). The solid line is the result of the best fit using Eq. (2).

the ethoxylation of the silica surface enhanced the Mb adsorption into the silica mesopore.

The volume fraction of $\mathrm{Mb}\left(f_{\mathrm{Mb}}\right)$ within the Ethoxy-MS was estimated from analysis of the reflection spectra by 4-layer Fresnel calculation. ${ }^{19}$ As typically shown in Fig. 4(a), the reflection spectra could be well-fitted to the calculation model, and the estimated $f_{\mathrm{Mb}}$ values for Cal-MS and Ethoxy-MS are 24 and 50\%, respectively. Miyahara et al. reported that maximum pore occupation of $\mathrm{Mb}$ in columnar silica mesopore of SBA-15 (pore diameter: $9.2 \mathrm{~nm}$ ) is $48 \%$ based on the adsorption model of a sphere-shaped protein. ${ }^{20}$ Hence, it can be considered that the ethoxylation of the inner pore surface is an effective way for the adsorption of $\mathrm{Mb}$.

In contrast to the efficient adsorption of $\mathrm{Mb}$ into the hydrophobic ethoxylated pore, the adsorption of $\mathrm{Mb}$ into the ODS-modified pore was significantly suppressed (Fig. 4(b)). One plausible reason for the suppression is the decrease of effective pore size by modification of long alkyl-chains of ODS on inner pore wall. However, since PEG-modified Mb can adsorb in the ODS-modified silica mesopore, ${ }^{14}$ it can be considered that the effective pore diameter of the ODS-MS layer is larger than the $\mathrm{Mb}$ size. The strong hydrophobicity of the ODS layer would be responsible for the suppression of the $\mathrm{Mb}$ adsorption into the pore.

In summary, we applied NPWG spectroscopy for studying adsorption behaviors of heme proteins (cyt $c, \mathrm{Mb}$ and HRP) into silica mesopores. The analysis of NPWG response provided apparent diffusion coefficient of a protein inside the mesopore.
The comparison of the apparent diffusion coefficients for heme proteins suggested that the diffusion of HRP inside the sizematched pore (pore diameter $=c a .6 \mathrm{~nm}$ ) was restricted due to the steric hindrance. When the protein size is smaller than the pore diameter, on the other hand, the electrostatic interaction between the protein and inner pore surface was proposed as a key factor to determine the protein diffusion. When influence of the surface hydrophobicity on the Mb adsorption was examined, it was confirmed that the maximum adsorption of $\mathrm{Mb}$ into the pore could be achieved by the ethoxylation of the inner pore surface. Since the NPWG spectroscopy provided kinetic information and rough quantification of the adsorption amount, it was useful to study adsorption of enzymes within inorganic nanoporous materials.

\section{Acknowledgements}

This work was supported in part by a Grants-in-Aid for Scientific Research (No. 22225003) from Japan's Ministery of Education, Culture, Sports, Science and Technology.

\section{References}

1. R. A. Sheldon, Adv. Synth. Catal., 2007, 349, 1289.

2. C.-H. Lee, T.-S. Lin, and C.-Y. Mou, Nano Today, 2009, 4, 165.

3. D. N. Tran and K. J. Balkus, ACS Catal., 2011, 1, 956.

4. C. Ispas, I. Sokolov, and S. Andreescu, Anal. Bioanal. Chem., 2009, 393, 543.

5. S. Hudson, J. Cooney, and E. Magner, Angew. Chem. Int. Ed., 2008, 47, 8582.

6. H. Arafune, A. Yamaguchi, M. Namekawa, Y. Sato, T. Itoh, R. Yoshida, and N. Teramae, Nat. Commun., 2014, 5, 5151

7. A. Vinu, M. Miyahara, and K. Ariga, J. Nanosci. Nanotechnol., 2006, 6, 1510.

8. M. Y. Chen and M. J. Sailor, Anal. Chem., 2011, 83, 7186

9. J. B. S. Ng, P. Kamali-zare, H. Brismar, and L. Bergstro, Langmuir, 2008, 24, 11096.

10. K. Nakatani, M. Miyanaga, and Y. Kawasaki, Anal. Sci., 2011, 27, 1253.

11. S. C. B. Gopinath, K. Awazu, M. Fujimaki, K. Sugimoto, Y. Ohki, T. Komatsubara, J. Tominaga, and K. C. Gupta, Anal. Chem., 2008, 80, 6602.

12. Y. Wang, C.-J. Huang, U. Jonas, T. Wei, J. Dostalek, and W. Knoll, Biosens. Bioelectron., 2010, 25, 1663.

13. K. Hotta, A. Yamaguchi, and N. Teramae, ACS Nano, 2012, 6,1541

14. H. Arafune, A. Yamaguchi, K. Hotta, T. Itoh, and N. Teramae, Anal. Sci., 2013, 29, 187.

15. T. Itoh, R. Ishii, T. Ebina, T. Hanaoka, T. Ikeda, Y. Urabe, Y. Fukushima, and F. Mizukami, Biotechnol. Bioeng., 2007, 97, 200.

16. A. Yamaguchi, H. Arafune, K. Hotta, T. Itoh, and N. Teramae, Anal. Sci., 2011, 27, 597.

17. S. Ong, X. Zhao, and K. B. Eisenthal, Chem. Phys. Lett., 1992, 191, 327.

18. S. T. Moerz and P. Huber, Langmuir, 2014, 30, 2729.

19. P. J. Cameron, A. T. A. Jenkins, W. Knoll, F. Marken, E. V. Milsom, and T. L. Williams, J. Mater. Chem., 2008, 18, 4304.

20. M. Miyahara, A. Vinu, and K. Ariga, Mater. Sci. Eng. C, 2007, 27, 232. 